\title{
Understanding How People Use Twitter During Election Debates
}

\author{
Katerina Gorkovenko \\ DJCAD \\ University of Dundee, UK \\ kgorkovenko@dundee.ac.uk
}

\author{
Nick Taylor \\ DJCAD \\ University of Dundee, UK \\ n.x.taylor@dundee.ac.uk
}

\begin{abstract}
During televised election debates, it is now common for citizens to take to social media to express their own opinions. Within this paper, we present a thematic analysis of the tweets generated during a debate from the 2015 UK General Election. The aim of this work is to explore the visible online behaviour seen on Twitter. We argue that by exploring what types of tweets emerge regardless of their political affiliation, we will gain a deeper understanding of citizen's behaviours online during live debates. We observed that citizens use Twitter to commentate along by sharing their opinions, experiences, make provocative or humorous statements, and interact and inform others.
\end{abstract}

Twitter, Political discourse, Social media, Second screens, UK General Election, Thematic analysis.

\section{INTRODUCTION}

The use of technology has transformed the way we consume and talk about politics. The recent 2016 US presidential election race was fought on multiple media platforms: as shocking stories about both candidates continuously dominated the news, the candidates themselves took to Twitter to defend themselves and attack each other publically. While the rough-and-tumble of politics is nothing new, social media allows the public to join in action. Televised political debates in particular are accompanied by vast amounts of viewer generated content (Anstead and O'Loughlin, 2011; Ausserhofer and Maireder, 2013; Bruns and Burgess, 2011; Kreiss, 2014). The public's reaction is visible on a wide range of social media sites, such as Facebook, Twitter and even Instagram (Mahoney et al., 2016; Shamma et al., 2009). Citizens use these opportunities to gauge the public's opinion, share their own opinion and enrich the experience of watching the debate (Gorkovenko and Taylor, 2016). This behaviour is called second screening.

Second screens are more broadly understood as personal devices used alongside television content for any related and unrelated purposes (Narasimhan and Vasudevan, 2012). Content producers have generated an array of applications to cater to this trend, such as series specific, event specific and check-in applications (Proulx and Shepatin, 2012). However, most viewers utilise existing apps, web browsers and social networks. Researchers in the area are especially interested in the online second screen activity, which is related to the debate. It is visible during the airing of different programs such as drama series (Nandakumar and Murray, 2014), live political events (Trilling, 2015), and reality television (Schirra et al., 2014). Viewers who use social media while watching television, report that it makes them feel part of a community and affirms their opinions (Schirra et al., 2014).

However, those benefits may not necessarily translate to second screening during political debates due to the fractious topics that are being discussed. Research in the area suggests that the tweets generated during debates are reactionary and evaluative (Shamma et al., 2009). For journalists and researchers this increase in publically available viewer generated data is an opportunity to access and evaluate the opinions of the public (Audderhofer and Maireder, 2013). This is often done though an array of computation analysis methods, such as sentiment analysis (Wang et al., 2012; Wang et al., 2011), word frequency counts and analysis of the networks of people (Shamma et al., 2009; Trilling, 2015). Sentiment analysis in particular often appears in the media as a predictor of popularity of the political candidates (Patterson, 2011). Although computer assisted analysis methods are convenient due to the vast quantity and speed of the generated content, the currently utilised political sentiment analysis tools have low accuracy rates, and only explore the broad positive or negative polarity of the text (Wang et al., 2012; Wang et al., 2011). 
Our research is motivated by the belief that by exploring visible content online using a qualitative analysis method, we can gain insight into the behaviours around second screening during political debates. This in turn can help us find ways to cater and enable citizens engage further with politics in order to empower citizens further to take an active role in political deliberation. The existing research into the use of Twitter in this context, includes analysis of who takes part in this discussion (Shamma et al., 2009); Trilling, 2015), how it is used by politicians (Kreiss, 2014), and what sentiment characterises the Tweets (Patterson, 2016). What is not well defined and understood is the array of behaviours and motivations visible in the tweets.

\section{RESEARCH OVERVIEW}

To understand why Twitter is used during televised political debates, we collected and analysed tweets during the 2015 UK General Election. Below, we describe the political context of these debates and our process of data collection and analysis.

\subsection{Research Context}

In 2015, televised debates were used for only the second time in a UK General Election. Although the first debates had been popular in 2010, there were long-running negotiations between government and the media over the format of the events. This led to speculation that they may not take place (Walker, 2015), as well as discussion over which parties should participate. At short notice, four separate events were set, two using a typical debate format and two using a Q\&A format with the individual party leaders. As might be expected, these debates were major social media events: the debate we studied received 1.5 million tweets during the two hours it aired (BBC News, 2015).

\subsection{Data Collection and Analysis}

Our analysis of Twitter use focuses on a single twohour debate, held on April $2^{\text {nd }} 2015$ on ITV. This was the only debate that featured all major parties and had a typical debate format. In addition to the three main parties, it also included four of the UK's smaller political parties. We have focused on Twitter due to its live event appeal and the ability to easily acquire tweets through a Twitter search API. Although it is far from being the only social media outlet utilised during televised debates, content is openly visible and broadcasters actively promote its use.

During the debate, a PHP script was used to access the Twitter search API every 30 seconds to request the 100 most recent tweets using \#leadersdebate, beginning at the start of the debate and continuing for approximately 90 minutes afterwards. This was the official hashtag for the debate and was actively promoted throughout the event. Retweets were excluded from the search in order to focus on original authored content. 38,569 tweets were gathered and $2 \%$ of these tweets (every 50th tweet captured) were used for an inductive thematic analysis, including any images in the tweets (Taylor and Gorkovenko, 2015). The data was coded manually by the lead researcher, after which the research team (made of two researchers) agreed upon a set of codes. Tweets that were representative of multiple codes retained all of the coded information until the themes were refined. The tweets were clustered together into the final themes, and could be allocated to one or more of the emergent themes.

\section{RESULTS}

Our analysis resulted in three major themes and another four large sub-themes. The theme of commentating, which included the sub-themes of sharing opinion, sharing experiences, humour and provoking; and the themes of interacting and informing. Described below, these themes cover a range of behaviours and give insight into the motivations that led the viewers to post.

\subsection{Commentating}

Commentary was the most prominent behaviour throughout the dataset. The content is diverse in nature, often quite humorous and juxtaposes the serious tone of the political debate. It is composed of four distinct sub themes: sharing opinion, sharing experience, humour and provokation.

\subsubsection{Sharing Opinion}

Although many tweets from the data set contained opinions, these tweets were overtly focused on personal evaluations of the debate and candidates. Twitter provides readers with evaluations of every aspect of the debate: the politicians' performance, the poll results, the debate structure, the audience, even the Twitter content itself. Most tweets guide the attention of the reader towards the negatives and positives of the debate ("brilliant entertainment so far - not sure it will change any voting choice. Pleased @natalieben has held her own this time”, “My \#leadersdebate scorecard at the three quarter mark: 1. Sturgeon 2. Wood 3. Clegg 4. Miliband 5. Cameron 6. Bennett 7. Farage").

Many ideas presented to the Twitter observer go beyond the events of the debate itself and take into account broader knowledge of politics and news ("Everything about treatment of Farage/ Bennett/ Sturgeon/ Wood would've been different if they had central [government] record to defend"). This evaluative process often merges with the endorsement and promotion of certain political leaders. Some simply show their support ("The women won hands down"), while others give detail about their position possibly in the hope to persuade 
others to vote in a similar way ("As a dad of 1 child with liver disease and 1 in [hospital] after huge spinal surgery there is only 1 party I trust for NHS \#labour").

\subsubsection{Sharing Experiences}

The viewers also annotate the debate with their personal experience of the event. Different aspects of the experience are addressed throughout the data set. For example, some share what is happening in their surroundings ("My mum got so tired of Nigel Farage's bullshit, she fell asleep halfway through the debate"), others express emotion ("Wish England could vote for @NicolaSturgeon" ^^^Just one of thousands l've seen tonight, wow, just wow!'). We also saw careful observations that guide the attention of the reader to intricate details ("Ed has gone from 'when' to 'if'. Clearly he feels he had a bad one"). Sharing personal experiences like the ones seen in this theme may help the social network mimic a physical community.

\subsubsection{Humour}

Humour manifests itself in a very complicated manner within the data. Although some of the tweets are jokes, it is difficult to separate them from other forms of commentary, as humour is usually combined with a political statement or opinion. For example, the tweet "So Conservative MPs all think Cameron won. Labour MPs all think Miliband won. Liberal Democrat MPs all went to bed early" gives an evaluation of the performance of the party leaders, but conveys this in a humorous way. Although, many tweets are just playful ("If you want a fun drinking game then do a shot every time ed millaband says 'and im sure people at home..."), sarcasm and satire are the predominant way that people express a humorous opinion ("Oh God it is immigration next. Farage is already salivating"). There seems to be an obvious desire to break up the seriousness of the debate-exemplified in the widespread use of memes (e.g. Figure 1). The images found throughout the data are characterised by their mocking and abusive nature. In addition to sharing the opinion of the author, this type of content had a secondary aspiration to either entertain, mock, or make opinions less antagonistic.

\subsubsection{Provocation}

Provocative tweets throughout the dataset have been written with a variety of intentions: some target at specific people or groups in an aggressive manner ("WANKER \#wallace \#EdMiliband"), while others try to provoke thoughts and reflections in the hopes of persuading others to vote for their choice of party ("So Cameron wants to take an already under performing NHS and improve it by stretching it over an extra 2 days"; "Vote Labour and get more borrowing, higher taxes and a greater financial burden on future generations. \#VoteConservative").

Most importantly, this theme shows how delicate and personal the topics in the debate can be. One of

\section{@Nigel_Farage is really being himself tonight. \#leadersdebate}

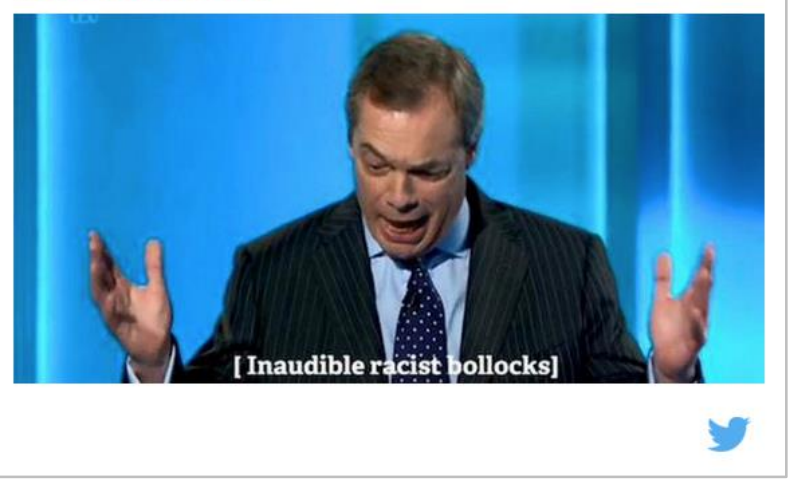

Figure 1: An example of a meme created during the live debate.

the most tweeted about moments from the debate was when a party leader with an anti-immigration stance condemned foreign HIV patients receiving free treatment in the UK (Dathan, 2015): this resulted in a large quantity of provocative commentary ("How can @Nigel_Farage @nigelmake such a statement about HIV very shameful"). Most tweets were a strong negative reaction to the statement and are provocative, emotional and intended to cause shame.

\subsection{Interacting}

Direct interactions between Twitter users were prominent during and after the end of the debate. This happened in two ways: the first was through direct replies to other users or tweets ("Rent to own scheme'. Isn't that a mortgage? this is what my 12 year old said!'), in the second users attempt to directly engage with a large segment of Twitter users rather than specific individuals ("Number geeks... If Plaid and SNP win every seat they stand in is it numerically possible for them to hold balance of power?"). Due to the way, the data was collected, only the individual tweets containing the debate hashtag were visible to the research team. The lack of access to the conversation threads limited data analysis and resulted in a lack of contextual information about the dialog that took place.

\subsection{Informing}

Users added additional layers of factual information to the broadcast through the use of links, images and text. During the debate this behaviour was characterised by its strong link to the current topic of conversation. However, while the content was factual, there was often a clear desire to sway the thinking of viewers in a particular direction ("In 2013 397,160 migrants were claiming benefits $(5.3 \%$ of the migrant population \& $7 \%$ of all benefit claimants)"). Near and after the end of the debate the informative tweets changed their purpose and 
were used to inform of news relating to the debate itself ("poll results - Guardian/ICM \#leadersdebate poll: 25\% Miliband 24\% Cameron 19\% Farage 17\% Sturgeon 9\% Clegg 3\% Bennett 2\% Wood"), or as a way to promote tools ("Feel more \#confused after \#leadersdebate? Here's some plain-English info on the issues that matter: http://t.co/s3ww2rM3og").

\section{DISCUSSION}

\subsection{Diversity of Behaviours}

Debate viewers used Twitter to commentate along by sharing opinions, experiences, post humorous and provocative tweets and interact with and inform others. Research suggests that debate viewers often use their second screens to gauge the public's opinions (Gorkovenko and Taylor, 2016). The diversity of behaviours we observed indicate that viewers can be exposed to a wide range of political views that may be absent from their other social networks. Research shows that $40 \%$ of Twitter users do not actively share their thoughts on the platform (Holt, 2013), while those who do report concerns about uncivil reactions (Bakker, 2013) and sounding ignorant (Semman, 2015). Thus, the provocative tweets visible online may be deterring some users from contributing during debates. Since more politically engaged individuals tend to have a curated experience on Twitter due to the pre-formed network of users they follow (Gorkovenko and Taylor, 2016), the provocative behaviour seen in the data may be a greater concen for less politically engaged viewers. These users may instead be drawn to Twitter due to the abundance of humour.

Although, some behaviours like sharing opinions, and humour may be boosting viewer engagement, others like provoking may be deterring users. Here the utopian vision of technology as a tool for political engagement and empowerment clashes with its use as a means for provoking and mocking. Future social networks for political engagement would benefit from exploring the relationship between observed behaviour and user engagement.

Social networks for political engagement around live debates may benefit from enabling users to modify their experience based on their preferred style of engagement. For example, users who are interested in adding value to the debate through entertainment could choose to see diverse but humorous content. Allowing viewers to immerse themselves in diverse commentry, which uses their preferred behavioral style, may in turn have the effect of encouraging them to post themselves.

\subsection{Taxonomy of Tweets}

Although sentiment analysis of Twitter has been the dominant method for studying discussion around political debates, we believe that the categorising of content based on token words may be an oversimplification of the content. As seen throughout the themes, the opinions and language used by the viewers is extremely subtle. Humour can often disguise negative attitudes, such as in the case of the tweet: "Lol Clegg talking about breaking promises", which alludes to the negatively perceived political track record of the leader of the Liberal Democrat Party. Such a statement may easily be misinterpreted using sentiment analysis tools. A future challenge for broadcasters and journalists is how to gain this sort of nuanced understanding of user created content across the vast volumes of data generated during a live debate.

The use of thematic analysis can not be implemented real time during a live debate. Instead we see several different opportunities for its future application. Categorising the behaviours visible on Twitter, which were generated during a debate, can illustrate the range of behaviour that can be observed online. These themes could then be used for the development of more specialised social networks around debates. Throughout the theme of commentating we saw that viewers shared not just their political views, but also their experiences and observations relating to the debate. This indicates that Twitter can be used as more than just a tool to evaluate the mood of the public, but also gain insights into the most noteworthy aspects of the debates. Furthermore, thematic analysis could be used to analyse in detail the views of the public regarding a political debate after it is aired, including the perceptions about the audience, participants, and questions asked by the host.

\section{CONCLUSION}

In this paper, we have contributed a more nuanced view of Twitter usage through a thematic analysis of tweets generated during a UK General Election debate. We found that debate viewers take to Twitter to share their opinions, experiences, post humorous remarks, provoke, inform and interact. These themes indicate that debate viewers access diverse opinion and content on Twitter during a live debate.

The next steps in gaining a fuller understanding of the online activity around political debates is to verify the above results through the thematic analysis of more debates from different countries. We believe that the behavioural themes on Twitter would be similar across the Western world, but might vary in other cultures. Future research into the online behaviours around political debates can then focus on different methods to understand the intrinsic motivations different users have for posting content.

\section{ACKNOWLEDGEMENTS}

This research was funded by a studentship from the EPSRC Doctoral Training Partnership. 


\section{DATA ACCESS STATEMENT}

The data set of Tweets used in this analysis is openly available from the University of Dundee repository (Taylor and Gorkovenko, 2015).

\section{REFERENCES}

Anstead, N. and O'Loughlin, B. (2011) The emerging viewertariat and BBC Question Time: television debate and real-time commenting online. The International Journal of Press/Politics 16, 4, 440-462. http://doi.org/10.1177/1940161211415519

Ausserhofer, J. and Maireder, A. (2013) National politics on Twitter: structures and topics of a networked public sphere. Information, Communication and Society 16, 3, 291-314. http://doi.org/10.1080/1369118X.2012.756050

Bakker, T. (2013) Citizens as Political Participants: The Myth of the Active Online Audience? PhD Thesis, University of Amsterdam. http://doi.org/11245/1.384371

BBC News. (2015) How the internet reacted to the leaders' debate. Retrieved January 18, 2016 from: http://www.bbc.co.uk/news/election-201532174120

Bruns, A, and Burgess, J. (2011) \#Ausvotes: how twitter covered the 2010 Australian federal election. Communication, Politics \& Culture 44, 2, 37-56.

Dathan, M. (2015) TV leaders' debate: 'You should be ashamed of yourself' Farage told as he lashes out at foreigners with HIV. Retrieved August 13, 2015 from http://ind.pn/1Gln95u

Gorkovenko, K. and Taylor, N. (2016) Politics at home: second screen behaviours and motivations during TV debates. Proceedings of the 7th Nordic Conference on Human-Computer Interaction (NordiCHI '16), 22. http://doi.org/10.1145/2971485.2971514

Holt, R. (2013) Twitter in numbers. Retrieved September 5, 2016 from http://www.telegraph.co.uk/technology/twitter/99 45505/Twitter-in-numbers.html

Kreiss, D. (2014) Seizing the moment: The presidential campaigns' use of Twitter during the 2012 electoral cycle. New Media \& Society 18, 8. http://doi.org/10.1177/1461444814562445

Mahoney, J., Feltwell, T., Ajuruchi, O. and Lawson, S. (2016) Constructing the visual online political self: an analysis of Instagram Use by the Scottish Electorate. Proceedings of the $2016 \mathrm{CHI}$ Conference on Human Factors in Computing Systems (CHI '16), 3339-3351. http://doi.org/10.1145/2858036.2858160
Nandakumar, A. and Murray, J. (2014) Companion apps for long arc TV series: supporting new viewers in complex storyworlds with tightly synchronized context-sensitive annotations. Proceedings of the 2014 ACM International Conference on Interactive Experiences for TV and Online Video (TVX '14), 3-10 http://doi.org/10.1145/2602299.2602317

Narasimhan, N. and Vasudevan, V. (2012) Descrambling the social TV echo chamber. In Proceedings of the 1st ACM Workshop on Mobile Systems for Computational Social Science (MCSS '12), 33-38. http://doi.org/10.1145/2307863.2307873

Patterson, D. (2016) Twitter sentiment analysis finds two candidates have never been more controversial-or unpopular. Retrieved January 10, 2016 from http://www.techrepublic.com/article/what-twittersentiment-analysis-is-saying-about-the-firstpresidential-debate/

Proulx, M. and Shepatin, S. (2012) Social TV: How Markets Can Reach and Engage Audiences by Connecting Television to the Web, Social Media and Mobile. John Wiley \& Sons. David A.

Semaan, B., Faucett, H., Robertson, S., Maruyama, M. and Douglas, S. (2015) Designing political deliberation environments to support interactions in the public sphere. Proceedings of the 33rd Annual ACM Conference on Human Factors in Computing Systems (CHI '15), 3167-3176. http://doi.org/10.1145/2702123.2702403

Schirra, S., Sun, H. and Bentley, F. (2014) Together alone: motivations for live-tweeting a television series. Proceedings of the SIGCHI Conference on Human Factors in Computing Systems ( $\mathrm{CHI}$ '14), 2441-2450.

http://doi.org/10.1145/2556288.2557070

Shamma, D., Kennedy, L. and Churchill, E. (2009) Tweet the debates understanding community annotation of uncollected sources. Proceedings of the First SIGMM Workshop on Social Media (WSM '09), 3-10. http://doi.org/10.1145/1631144.1631148

Taylor, N. and Gorkovenko, K. (2015). Tweets from \#leadersdebate (2015 UK General Election TV Debates). https://doi.org/10.15132/e0f2d122f40f-449d-93b1-e58300ab1f3f

Trilling, D. (2015) Two different debates? Investigating the relationship between a political debate on TV and simultaneous comments on Twitter. Social Science Computer Review 33, 3, 259-276.

http://doi.org/10.1177/0894439314537886 
Walker, P. (2015) General election 2015: the drama behind the leaders' debates. Retrieved August 12, 2015 from: http://gu.com/p/46pph/sbl

Wang, H., Can, D., Kazemzadeh, A., Bar, F. and Narayanan, S. (2012) A system for real-time Twitter sentiment analysis of 2012 U.S. presidential election cycle. In Proceedings of the ACL 2012 System Demonstrations (ACL '12), $115-120$
Wang, X, Wei, F., Liu, X., Zhou, M. and Zhang, M. (2011) Topic sentiment analysis in twitter: a graph-based hashtag sentiment classification approach. In Proceedings of the 20th ACM international conference on Information and knowledge management (CIKM '11), 1031-1040. http://dx.doi.org/10.1145/2063576.206372 